\title{
A New Wavelet Denoising Method for Noise Threshold
}

\author{
Sureewan Jangjit ${ }^{1, \mathrm{a},{ }^{*}}$ and Mahasak Ketcham ${ }^{2, \mathrm{~b}}$ \\ 1 Department of Information Technology, Faculty of Information Technology, King Mongkut's University \\ of Technology North Bangkok, 1518 Pibulsongkram Road, Bangsue, Bangkok 10800, Thailand \\ 2 Department of Information Technology Management, Faculty of Information Technology, \\ King Mongkut's University of Technology North Bangkok, 1518 Pibulsongkram Road, Bangsue, Bangkok \\ 10800, Thailand \\ E-mail: as5607011956013@email.kmutnb.ac.th (Corresponding author), bmahasak.k@it.kmutnb.ac.th
}

\begin{abstract}
This study is proposed a new adaptive threshold based on noisy speech for each sub-bands with low complex and it is suitability for range of human hearing and range of hearing test. A new method is used wavelet 1-D experimental signal for denoising. It provided the optimal adaptive threshold of three sub-band with applies to the detail coefficients. The speech enhancement is used of threshoding on the adpated wavelet coefficients, and the results are compared a variety of noisy speech and four well-known benchmark signals. The results, measured objectively by Signal-to-Noise ratio (SNR) and Mean Square Error (MSE), are given for additive white Gaussian noise as well as two different types of noisy environment. The new method called Adaptive Thresholding with Mean for hybrid Denoising method of hard and soft function (ATMDe) and applied to hearing loss and it is found that it increases the signal-to-noise ratio by more than $114 \%$ and decreases the mean-square-error (MSE). The result of new method with SNR and MSE is higher than standard denoising methods. Hence, the new method was found that has good performance and adaptive threshold value is better than other methods.
\end{abstract}

Keywords: Wavelet denoising, noise threshold, noise reduction, adaptive threshold, speech enhancement.

ENGINEERING JOURNAL Volume 21 Issue 7

Received 16 February 2017

Accepted 3 May 2017

Published 29 December 2017

Online at http://www.engj.org/

DOI:10.4186/ej.2017.21.7.141 


\section{Introduction}

The objective of speech enhancement were to improve quality and intelligibility of speech [1]. The main problem of speech distort is noise. They are main problem that will affect speech understand and an important problem of speech and signal processing [2]. This is highly dependent on how much noise is removed. The different approaches have been proposed to solve this problem and audio signals include speech is no models availability [3].

The currently, the popular reduce noise methods are divided two methods: noise reduction and denoising. There are increased performance of speech and sound quality. The main problem of them is clearly sound noisy environment [4]. First, noise reduction is reduced noise of input sound that qualities depend on noisy environment database. The advantage is output sound quality if noisy environment of input sound is match with noisy environment of output sound but there is not match because of many of noisy environment cause output sound is distort. This method is not support small device [5] such as hearing aid because the noisy environment database is large that it want store area for keep its. Second, denoising is most reduced noise. The important of variable is threshold. If high threshold affect low SNR and high distort, on the other hand if low threshold affect high SNR and low distort but it is not reduce noisy environment. This advantage of denoising didn't use to noisy environment database and low complexity. The important of this method is selected threshold. There is more value of threshold and it not answer for optimal anyone threshold values. Furthermore, these researches are used low complexity method for finding threshold and denoising depends on attribute of signal. The most famous of solve this problem with denoising approaches are wavelet thresholding and spectral subtraction [6]. The denoising method is used wavelet transform and wavelet thresholding algorithm for reduce noise [7, 8]. There are modified of the hard thresholding [8] and soft thresholding [7].

In addition, other problem with current wavelet denoising is the number of decomposition levels for denoise, and alternative wavelet because they have the flexibility to adjust the thresholds [9]. Many researches have evaluated result of denoising with signal-to-noise ratio [2,10], segmental signal-to-noise ratio [2,11, 12], Mean Squared Error (MSE) [7, 13], and used realistic noisy environment [2, 8, 11-13]. Furthermore, the denosing method is applied to various kinds for example $[13,14]$ proposed denoising base on wavelet and [8] are proposed new speech enhancement system using wavelet thresholding algorithm with modified of the hard thresholding function which sets the coefficients of wavelet to zero when lower than the threshold value because of time-frequency discontinuities in the enhanced speech spectrum and used wavelet was "db8". There are evaluated sound of speech under white Gaussian noise, pink noise, [10] is proposed a novel thresholding function base on wavelet is more elastic than the soft and hard thresholding. The MATLAB simulation of signal processing area results show that gives better SNR gains than DJps hard and soft threshold methods and suitable with a low SNR in many signals, and multi-talker babble noise base on a posteriori segmental SNR for each sub-band for remove more noise from the sub-bands. These systems are used signal-to-noise ratio (SNR) and the automatic speech recognition (ASR) accuracy for the performance of speech enhancement algorithm, and voice activity detector (VAD) permits difference between the speech and the non-speech segment because it is robust to the noisy context $[15,16]$. This result show that the ASR reduced of the noise and increase the recognition rate in noisy environment, [12] proposed estimated a nonnegative spare coding with a combined dictionary of the spectrograms of clean signal and noise and presented using synthetic (chirps) and real signal with additive noise. This method is evaluated measures: the perceptual evaluation of speech quality and the segmental signal-to-noise ratio. This results show that method improved the quality of the signals, mainly under severe degradation, [11] use the segmental SNR (SegSNR) to determine the gain factor of wavelet coefficient threshold (WCT) can efficiently remove the background noise (noisy speech is additive colored-noise). This method is used noise masking threshold (NMT) of each wavelet subband for adjust WCT can be perceptually mask residual noise and reduce speech distortion, [2] the new method of speech signal enhancement is used Bionic wavelets transform and its incorporated Giguere-Woodland auditory model. The result shows that the performance is competitive with some of the best methods in signal processing field, and in addition, $[17,18]$ proposed new ECG denoising base on noise reduction algorithm with to perform windowing in the emprical mode decomposition (EMD) and discrete wavelet transform (DWT) domain. The simulation show that this method is reduced noise from the noisy ECG signal. Moreover, the example is relevant to the denoising in other topic such as healthiness for example [7] is improved wavelet threshold denoising method for reduce the noise in the heart sound signal, and the example is relevant of speech such as [19] propose segment-based speech recognition framework is increased 
the phoneme recognition accuracy about $25 \%$ of the one obtained from the baseline segment-based speech recognition.

This new method is presented to denoise 1-D using wavelet transforms because of it is the state-of-theart wavelet denoising method [9] and improved the hard and soft wavelet threshold denoising with idea of the parameter is used to change the size of the deviation between reconstruction signal and the original signal. These measurement performances are SNR and MSE. The simulation shows that this improved method is superior to by hard and soft wavelet threshold denoising method. In addition, the computational auditory scene analysis (CASA) method is used major parameter with the interaural intensity difference (IID) for denoising. The simulation show that the SNR of the heart sound is improve after denoising [20], [21] proposed an automated multiband denoising scheme for improving the quality of auscultation signals against heavy background contaminations. This method is adapted subtraction scheme in the spectral domain with processes each frequency band in a non-uniform. Many relevant research areas of denoising is found that the one popular method and the most of them are not tested real noisy environment but there are evaluated sound under white Gaussian noise and color noise similar real noisy environment. Thus, the feature of issue 1: new method is low complex. It is considered high power spectral density. The feature of issue 2: new denoising algorithms is each threshold for each sub-band depends on attribute of signal that it is relevantly human hearing range and it will be applied to hearing aid for hearing loss.

In this paper, a new wavelet denoising approach is a new finding threshold and new denoising threshold base on wavelet shrinkage. The result show that significantly improves denosing compared to other standard wavelet and previous methods. It used for denoising experimental signal. The rest of the paper is organized as flows. First, the wavelet shrinkage denoising method is provided. Second, the issues of new finding thresholding and new denoising threshold are discussed. Third, an analysis of noise is presents. Fourth, the new proposed method is presented. Five, examples of denoising using experimental results are presented and compared with other wavelet denoising methods. Finally, conclusion and discussion is given and future extensions.

\section{The Wavelet Shrinkage Denoising Method}

The wavelet transform is analyzed signals. This study is used wavelet transform because of simple and naive for denoise represented of single level discrete 1-D wavelet transform that is the state-of-the-art wavelet denoising [9]. This method has become common signal processing technique in different area and the result indicate that wavelet functions Daubechies 44 (db44) provides a better fit to tested biosignals [22] but experiment of this study is found that the Coiflets 1 wavelet is better suited in this study. In addition, the wavelet transform has powerful tool because this process is removed the noise of signal and image [14].

The transform method is two processes consist of decomposition or analysis and reconstruction or synthesis. The decomposition process is decomposed signal. The reconstruction process is reconstructed signal. This study is used criteria of composition of noise frequency in environment of areas of daily living for student with hearing loss. The human hearing is the range of $20 \mathrm{~Hz}$ to $20 \mathrm{kHz}$ that are produced audible the sound to human ear and hear sound best is range $1 \mathrm{kHz}$ to $4 \mathrm{kHz}$ [23] but experiment of this study is found that the most of power spectral density is range $500 \mathrm{~Hz}$ to $4 \mathrm{kHz}$. Thus, this study is used them. This is one of the most important features. One solution of this way is kept feature value for each node calls the wavelet coefficients $[24,25]$ is proposed threshold wavelet coefficient and recover signal $\mathrm{S}(\mathrm{x})$ from noisy $\mathrm{N}(\mathrm{x})$, where $\mathrm{i}=1,2, \ldots, \mathrm{N} ; \mathrm{S}(\mathrm{x})=$ signal; $\mathrm{N}(\mathrm{x})=$ noisy of signal, and $\mathrm{b}_{\mathrm{i}}=$ Gaussian white noise see in Eq. (1)

$$
N(x)_{i}=S(x)_{i}+b_{i}
$$

The one of reduce method is wavelet denoising. This method is divided three main steps [14]: First step, decompose or the noisy signal is transform into wavelet domain. Second step, the wavelet coefficients is applied threshold value. This step is an important because the threshold value is applied to remove noise with thresholding function. The selections of the threshold value affect quality of signal because the large threshold is selected. This result is a loss of useful information and speech is most of distortion but the small is selected. This result does not remove the noise. The many researches $[8-10,13,14]$ is used The soft and hard threshold of wavelet denoising because it low complex and short processing times but the main problem of this method is finding optimal threshold that is adaptive threshold for response to the noisy environment. Third step, 
reconstruct or the inverse wavelet transform is transformation of denoising signal into time domain using the original approximation coefficients and the modified detail coefficients.

The best answer is high SNR. The solutions of it are a detail wavelet coefficient is either a signal or a noise coefficient that the hard thresholding is better suited, whereas it is both signal and noise that the soft thresholding is better suited and the wavelet shrinkage method better signal denoising with minimum computation complexity [9] and wavelet shrinkage denoising algorithm (see Table 1 and Fig. 1) where $\mathrm{S}(\mathrm{x})$ is a noisy speech of input signal, $D_{i}$ is a Detail wavelet coefficient of noisy speech where $i=1,2, \ldots, n, A_{i}$ approximate coefficient is where $\mathrm{i}=1,2, \ldots, \mathrm{n}$, and the $\mathrm{S}\left(\mathrm{x}^{\prime}\right)$ is noisy speech of output signal.

Table 1. Wavelet shrinkage denoising Algorithm [9].

wavelet shrinkage denoising

1: $\quad$ Select wavelet

2: Select $\mathrm{k}(1 \leq \mathrm{k} \leq \mathrm{N})$ decomposition level for denoising of the coefficient Detail $\left(\mathrm{D}_{\mathrm{i}}\right)$ where $\mathrm{i}=1,2, \ldots, \mathrm{n}$, when $\mathrm{N}=\left\lfloor\log _{2}(\mathrm{~L})\right\rfloor$, and $\mathrm{N}=\operatorname{length}(\mathrm{x})$ that $\mathrm{x}$ is discrete noisy speech

3: Compute decomposition of discrete wavelet transform (DW'T) with the $\mathrm{k}^{\text {th }}$ level, so the output of this step is $\mathrm{k}$ Detail components and $\mathrm{k}^{\text {th }}$ approximate components

4: Calculate the noise threshold for $\mathrm{k}$ Detail components

5: Apply noise threshold to the k selected detail component

6: Inverse DWT of $\mathrm{k}$ Detail components, and approximate components

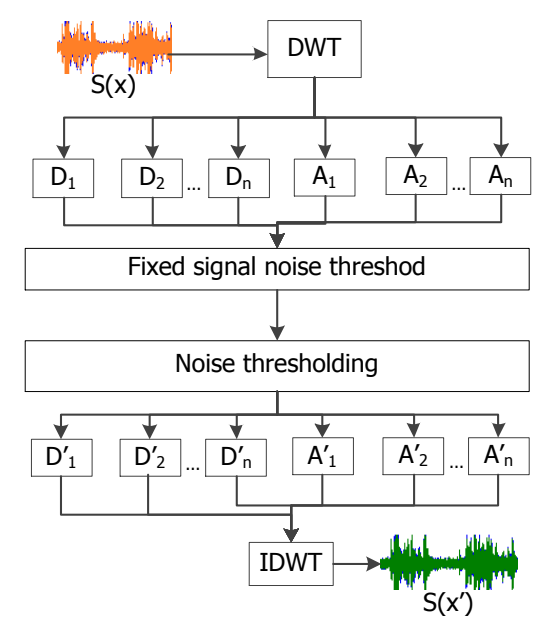

Fig. 1. Block Diagram of standard wavelet Shrinkage method [9].

A limitation of shrinkage methods is fixed threshold of method was not adjusted. If adjusting threshold enable users to reach optimal thresholds for signals. And the definition way of estimate noise value $\sigma^{\text {Noise }}$ is not clear due to the choice of $\sigma^{\text {Noise }}$ greatly influences the noise threshold thr. In addition, the popular threshold function for wavelet denoising is hard threshold function depicted in Eq. (2). It is better suited when a Detail wavelet coefficient is either noise or signal coefficient but the defect of us was discontinued at the threshold point that it will be lead to severe oscillation and limit its further application [26], and soft threshold function depicted in Eq. (3). It is better suited coefficient that are include both noise and signal [9].

The hard threshold,

$$
w_{T}= \begin{cases}w & |w| \geq t h r \\ 0 & |w|<t h r\end{cases}
$$


The soft threshold,

$$
w_{T}= \begin{cases}{[\operatorname{sign}(w)](|w|-t h r)} & |w| \geq t h r \\ 0 & |w|<t h r\end{cases}
$$

where $\mathrm{w}$ means the wavelet coefficients value, $\mathrm{w} T$ is the wavelet coefficients value imposed threshold, thr is threshold value, and $|\mathrm{w}|$ is absolute value of wavelet coefficients, respectively.

\subsection{Measurements}

This section describes measure for performance that Speech quality measures use speech degraded by noise. The one of important for evaluated off-line, there are used to compare an algorithm with alternatives or to evaluate the use of different probability models within an algorithm [1]. There are two types of distortion and quality measure: object measures for example signal to noise radio (SNR) and MSE. This study is used two methods of object measures are SNR and MSE for verifier the results. There are the following: the performance of signal have measured with Signal to Noise ratio (SNR) that the most used distortion measures and measure for quality for speech signals [1]. The both measure are computed and compared that the high SNR denotes the quality of sound more than low SNR and the low MSE denotes the quality of sound less than high MSE. So, the results of this experiment are compared with other standard method. Moreover, the threshold is usually selected to highly the SNR for given high quality of signal that SNR is defined as Eq. (4), and Mean Square Error (MSE) is used to evaluate performance of an estimator. This method is also useful to regard accuracy in statistical estimation, precision, and bias concept.

The minimum MSE is best estimator and good performance of this study because of the similarity between output signals and reference signal, MSE is depicted in Eq. (5).

$$
S N R=10 \log _{10}\left(\frac{P_{\text {signal }}}{P_{\text {noise }}}\right) d b
$$

where $P_{\text {signal }}$ and $P_{\text {noise }}$ are the power of signal and noise respectively.

$$
M S E=\sum_{i=1}^{n} \frac{\left(r f^{i}-o u t^{i}\right)^{2}}{n-1}
$$

where $r f$ is reference signal, out is output signal and $i$ is samples in dataset of size $n$.

$$
\begin{gathered}
\mu=\frac{\sum_{i=0}^{N-1} x_{i}}{N} \\
\sigma=\sqrt{\frac{\sum_{i=0}^{N-1}\left(x_{i}-\mu\right)^{2}}{N}}
\end{gathered}
$$

The mean, indicated by $\mu$ that it for average value of a signal that index $\mathrm{i}$, run from 0 to $\mathrm{N}-1$ and $\mathrm{N}$ is sum the values in the signal defined as Eq. (6). The standard deviation denoted by $\sigma$ (sigma) that it refers to how the signal fluctuates around the mean value defined as Eq. (7). This study was used them for new denoising algorithm at issue 1 that it considered the coefficients in range of denoising.

\subsection{The Data and an Analysis of Noise}

The objective of this section is to analysis noisy environment in areas of daily living for student with hearing loss with range $20 \mathrm{~Hz}$ to $20 \mathrm{kHz}$. The finding of it is intensity of frequency. The computation was calculated in MATLAB on a 32 bit operating system with Intel(R) Core(TM) i5 CPU @2.67GHz, RAM 4 GB. The noise was used with SONY device model ICD-UX512 that recorded of sound with mono, sampling rate $48000 \mathrm{~Hz}, 16$ bits, mono, sound length 20 seconds.

The data is divided into two sets including: First, the data came speech corpus (NOIZEUS) [27] including clean speech, noisy speech in different environments with SNR level $0 \mathrm{~dB}$ and $10 \mathrm{~dB}$. Second, the data is my recorded in noisy environment. In addition, the each set data of them are divided into two types: type 1 
indoor noise, this type is sound within a building. There is often used such as sound in class room, meeting room, library and canteen. The most of indoor noises are air condition and soft speech; type 2 outdoor noise, this type is outside a building. There is frequency used such as besides building, road side, in car. The most noises are wind, bird sound, sound of motorcycle and speech. This experiment was carried out to analyze the intensity of spectrum. We identify contours by difference region. The orange region is high intensity of noise see details in Fig. 2. The result show that spectrum, wave and contour of type 1 and type 2 . The type 1 was found that the most of this type was found that the intensity in low frequency less than $20 \mathrm{~Hz}$ with mean about $-7.166 \mathrm{~dB}$. The graphs are for example in spectrum, wave, and contour (see Fig. 2).

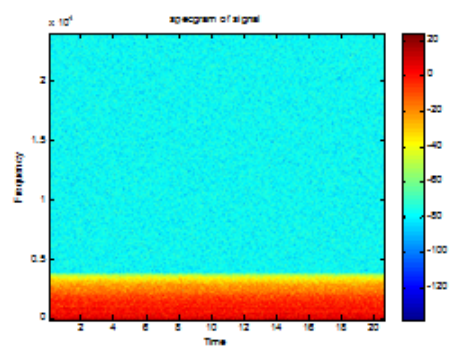

(A) spectrum

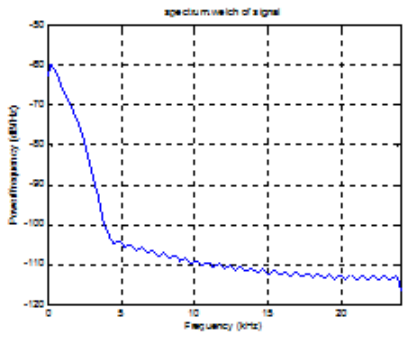

(B) Wave

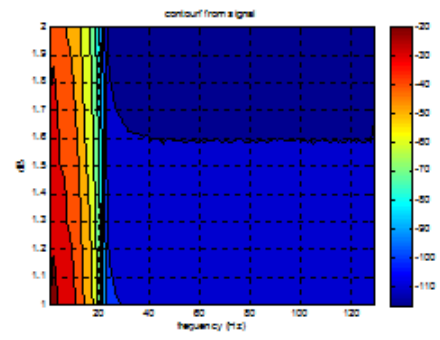

(C) Contour

Fig. 2. Indoor noise environments.

The type 2 was found that the intensity in low frequency less than $90 \mathrm{~Hz}$ with mean about $-2.775 \mathrm{~dB}$. The graphs of indoor noises are similarly in spectrum, wave, and contour; see in Fig. 3.

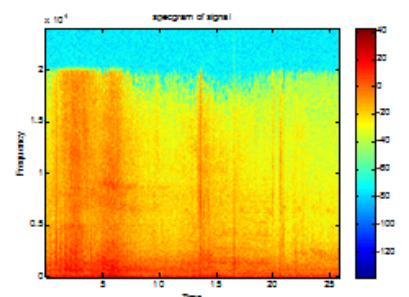

(a) Spectrum

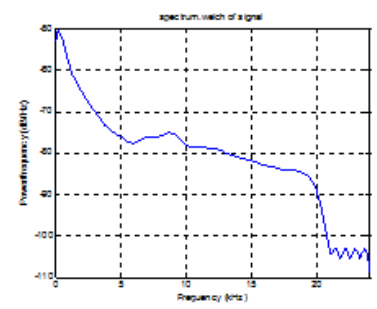

(b) Wave

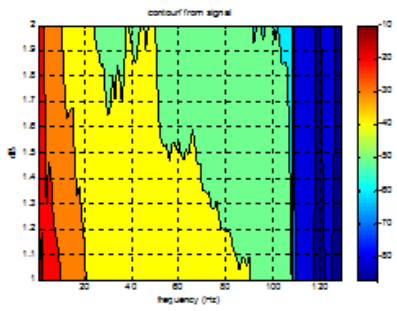

(c) Contour

Fig. 3. Outdoor noise environments.

Based on the above experiment issues, the condition of filter signals of type 1 was filtered $\mathrm{dB} \leq-4 \mathrm{~dB}$ at signals frequency less than $20 \mathrm{~Hz}$ but type 2 was filtered $\mathrm{dB}$ at signal frequency less than $90 \mathrm{~Hz}$, therefore this process reduced noisy environment and the results of this experiment compared between type 1 and type 2. The original noisy speech did not do pre-processing and on the other hand, the new noisy speech did do pre-processing the original noisy speech is result of sound. These results showed that the SNR and dB of new noisy speech is highly than the original noisy speech, so the new noisy speech is more loudness and clearly sound.

In addition, The data are more used in this study includes the noisy environments of areas of daily living for student with hearing loss and a noisy speech corpus (NOIZEUS) [27]. There were analyzed with the power spectrum. It is the distributions of the signal power various frequencies. It can be analysis of residual signal which is difference between original signal and noise signal. This method used to deduce the existence of repetitive structures in the signal process. The power is concentrated in one frequency.

\section{New Proposed Method}

The new proposed method base on the wavelet shrinkage is applied to hearing aid for hearing loss. It is used threshold for each sub-band that relate human hearing range and hearing test range are displayed in the form of an audiogram. The range of human hearing is between $20-20,000 \mathrm{~Hz}$ and this study is related to speech of 
human. It has improved the effect of speech quality signal denoising and adapted with hearing loss who worn hearing aids. The hearing loss is being tested listens to sound with the audiogram which is measuring loudness. The frequency of audiogram is generally used 250,500, 1000, 2000, 4000, and $8000 \mathrm{~Hz}$. Hence, the range of human hearing and frequency of audiogram are considered in this study. It intended for filter ranges three ranges that is divided including: first, less than $500 \mathrm{~Hz}$ is defined $20-250 \mathrm{~Hz}$ or $90-250 \mathrm{~Hz}$. This either range is selected as condition of noise environment. It is depended on indoor noise filter is $20-250 \mathrm{~Hz}$ or outdoor noise filter is $90-250 \mathrm{~Hz}$. It called background signal; second, $500-4000 \mathrm{~Hz}$ is human hearing is most sensitive with speech in this range and this experiment of noisy environment. It called foreground signal; third, more than $4000 \mathrm{~Hz}$ is defined $4000-20000 \mathrm{~Hz}$ that is relevant human hearing. It called background; see in Fig. 5 .

\subsection{Threshold Consideration}

The many research were used the wavelet thresholding for speech enhancement [8]. The main objective of this work is to propose a new wavelet threshoding algorithm based on the adaptive threshoding with low complexity and to check property of signal that it is suitable for any threshold function (see detail the section 2). Moreover, the detail coefficient is high frequency that it was denoising but it really listened to understand, whereas an approximate coefficient is low frequency that it is important of speech. If it was denoising that it distort, it does not listened to understand. Thus, this study is selected detail coefficient for denoising on foreground signal and background signal see in Fig. 5.

The new method is calculated mean of signal for thresholding see in Eq. (6). The currently, this method was not implemented for finding threshold of wavelet denoising. It is applied to three sub-bands: foreground and background that prepared for next step of denoising.

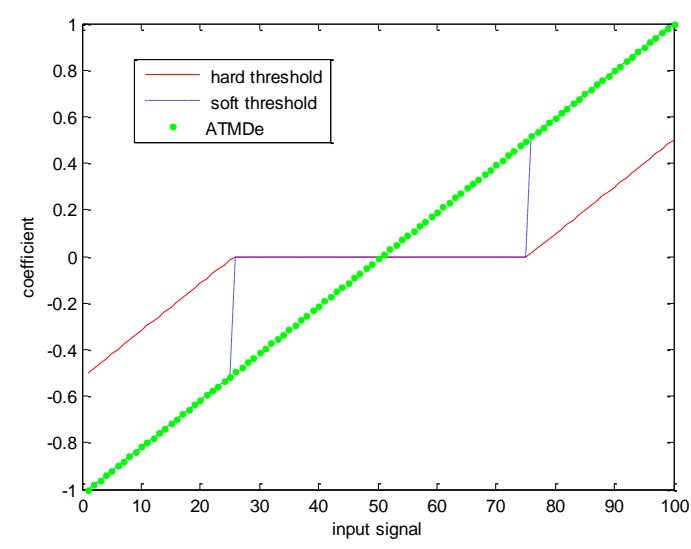

(a) Overlap threshold function
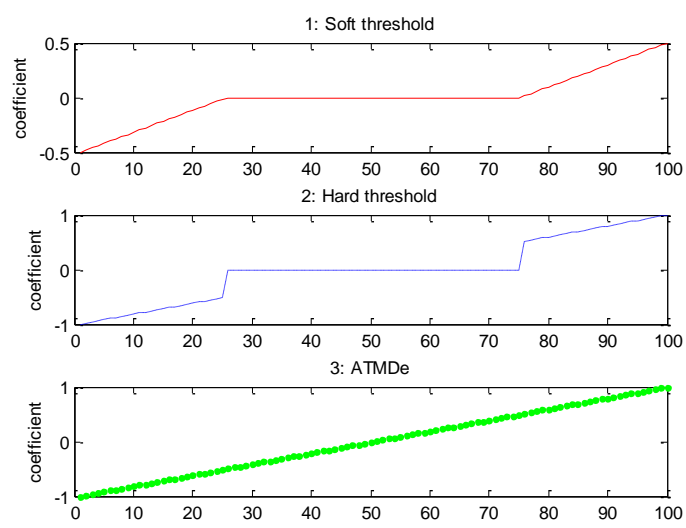

(b) Sequence threshold function

Fig. 4. The figure is center-aligned and the caption of the figure is left-aligned.

Figures 4 (a) and 4 (b) show denoising results on hard thresholding with red line, soft thresholding with blue dash line and green dot line respectively. The hard and soft thresholding are zero when coefficient is low than threshold but the proposed method is slowly increased that it depend on the property of signal.

\subsection{New Denoising Algorithm}

The solutions of denoising method is considered attribute of signal in each sub-band that it are a detail wavelet coefficient is either a signal or a noise coefficient is used hard thresholding is better suited, whereas it is both signal and noise is applied soft thresholding is better suited [9].

The main ideas of new proposed: First, pre-processing is analyzed signals for filter of noisy environment; Second, signal is divided to three sub-bands for adjust wavelet coefficients for freedom of threshold with low complexity method. It can be reliably used foreground range for denoising experimental signals (see Fig. 5). The other reason is that this ranges are related Pure tone of hearing human and this study will be apply with hearing aid. In addition, the background range is excluded foreground range. This range mostly composed sound of environments, and finally, new denoising algorithm is reduced noise follow up threshold. The new 
proposed called Adaptive Thresholding with Mean for hybrid Denoising method of hard and soft function (ATMDe) in Table 2.

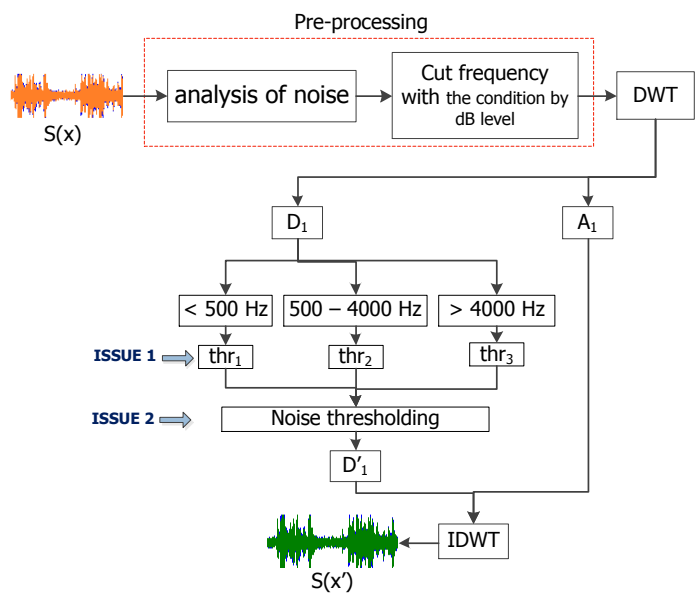

Fig. 5. Block Diagram of ATMDe.

Table 2. Mean for hybrid Denoising method of hard and soft function (ATMDe) algorithm.

ATMDe algorithm

1: $\quad$ Select noisy speech of signal

2: Pre-processing with the condition of filter that indoor (type 1) was filter signals frequency less than $20 \mathrm{~Hz}$ and outdoor (type 2) was filter signals frequency less than $90 \mathrm{~Hz}$ and

3: $\quad$ select wavelet

4: Decomposition 1 levels to denoise the detail coefficient with discrete wavelet transform (DWT), and $\mathrm{N}=$ length $(\mathrm{L})$ where $\mathrm{L}$ is the discrete noisy speech

5: Filter of signal is three sub-bands : $<500 \mathrm{~Hz}, 500-4000 \mathrm{~Hz}$, and $>4000 \mathrm{~Hz}$

6: Calculate equation 6 for $\mathrm{thr}_{1}, \mathrm{thr}_{2}$, and $\mathrm{thr}_{3}$ of each sub-bands, respectively (ISSUE 1)

7: Calculate denoising (ISSUE 2)

7.1: Select each sub-band signal

7.2: Take sub-band signal coefficient of the detail coefficient

7.3: Calculate equation 8

7.4: Apply noise thresholding to detail coefficient

8: Take inversed discrete wavelet transform (DWT) of the result detail coefficient and approximate coefficient

$$
w_{T}= \begin{cases}\text { coeff } & :\left(\text { coeff } \leq \mu_{\text {sub }}+\sigma_{\text {sub }}\right) \text { and }\left(\text { coeff } \geq \mu_{\text {sub }}-\sigma_{\text {sub }}\right) \\ \text { coeff } \times \mid \text { coeff } \mid-t h r & :\left(\text { coeff }>\mu_{\text {sub }}+\sigma_{\text {sub }}\right) \text { and }\left(\text { coeff } \leq \mu_{\text {sub }}+2 \sigma_{\text {sub }}\right) \\ & \text { or }\left(\text { coeff }>\mu_{\text {sub }}-\sigma_{\text {sub }}\right) \text { and }\left(\text { coeff } \leq \mu_{\text {sub }}-2 \sigma_{\text {sub }}\right) \\ 0 & \text { : otherwise }\end{cases}
$$


where $w_{T}$ is denoised wavelet coefficients, coeff is transformed coefficient, thr is thresholding of sub-band signal and $\mu_{\text {sub }}$ is mean of sub-band signal depicted in Eq. (6), and $\sigma_{\text {sub }}$ is standard deviation of sub-band signal depicted in Eq. (7). So, in Eq. (8) is designed of denoised wavelet coefficients depicted in Fig. 6 that is selected cD:Details because it is details of high frequency. The sound speech was removed of high frequency that even though it distorted, it really listened to understand. On the other hand, the sound speech was removed of low frequency of cA:Approximate. It is important for human speech. Even though it distorted, it does not listened to understand. Consequently, the threshold is selected and applied threshold to the detail coefficients. It is significantly provided optimal threshold. It was related to develop wavelet threshold of denoise for noisy environment base on section 3. Furthermore, this study has applied new method thresholding in new denoising algorithm for reduce noise. It has been investigated and the most promising result proposed that significantly improves the SNR of denoised signal.

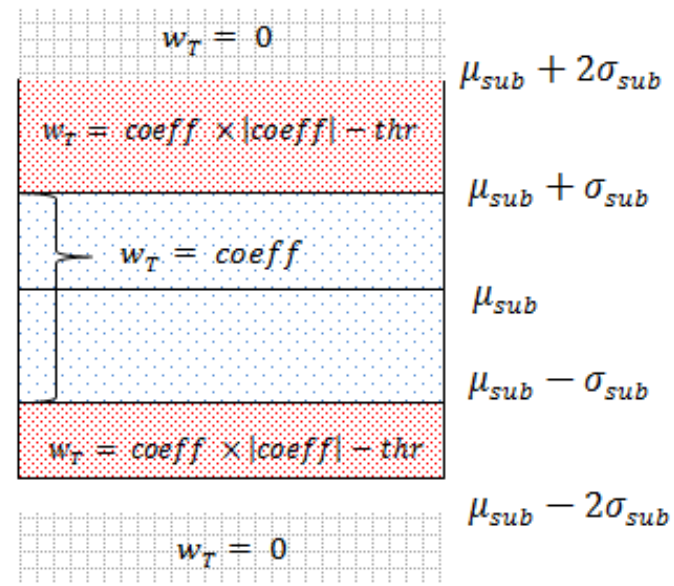

Fig. 6. Design of denoised wavelet coefficients.

\section{Examples of Denoising}

The problem of wavelet shrinkage is fixed threshold and we need to apply to hearing loss. Hence, this study solved with a new adaptive threshold for each sub-bands with low complex and it is suitability for range of hearing test. This experiment was carried out to test the accuracy of New proposed and another method. To evaluate the effectiveness of using new proposed called ATMDe for enhancement of noisy speech, we compare it to other standard approaches on this section. These results shown in Table 3-8 are compared using SNR (dB), SNR, MSE, and Time (seconds) indices of mean that the experiment are 6 examples including: First, example 1 and Table 3 with clean that they used data in [27]. Second, example 2 and Table 4 with noisy speech and noisy environment that used in [28] for research and came from [27]. Third, example 3 and Table 5 are used data from my recoded of environment that is described in section 2.2 within noisy speech and noisy environment. Fourth, example 4 and Table 6 are used data in [27] for compared SNR input and SNR output. Fifth, example 5 and Table 7 are used data form my recorded of environment and Sixth, example 6 and Table 8 are compared new proposed versus four well-known benchmark signals. So, for each experimental, the method that obtained the best denoising quality is emphasized in bold-face.

Table 3. Example 1: Signal to Noise Ratio (SNR): Comparison of ATMDe with other standard wavelet in clean speech.

\begin{tabular}{lcccc}
\hline \multirow{2}{*}{ Signal } & \multicolumn{4}{c}{ Clean } \\
\cline { 2 - 5 } & SNR $(\mathrm{dB})$ & SNR & MSE & Time \\
\hline Minimaxi-hard & 66.7905 & 2315.6667 & $2.20 \mathrm{E}-05$ & 0.3587 \\
Minimaxi-Soft & 66.7905 & 2315.6667 & $2.20 \mathrm{E}-05$ & 0.3587 \\
SUREShrink-Hard & 66.7905 & 2315.6667 & $2.20 \mathrm{E}-05$ & $\mathbf{0 . 0 8 8 3}$ \\
SUREShrink-Soft & $\mathbf{6 6 . 7 9 0 5}$ & 2315.6667 & $2.20 \mathrm{E}-05$ & $\mathbf{0 . 0 8 8 3}$ \\
ATMDe & $\mathbf{6 7 . 9 4 9 6}$ & $\mathbf{2 6 6 6 . 2 0 0 0}$ & $\mathbf{1 . 7 3 E - 0 5}$ & 0.1452 \\
\hline
\end{tabular}


The results in the ATMDe with other standard wavelet in clean speech are summarized in Table 3 . The SNR of ATMDe was the highest with SNR 15.14\% and MSE 27.17\%. In contrast, the processing time is slower than the both SUREShirnk but it is quickly than the both Minimaxi.

Table 4. Example 2: Signal to Noise Ratio (SNR): Comparison of ATMDe with other standard wavelet in Noisy speech and noisy environment.

\begin{tabular}{l|cccc|cccc}
\hline \multirow{2}{*}{ Signal } & \multicolumn{4}{|c|}{ Indoor } & \multicolumn{4}{c}{ Outdoor } \\
\cline { 2 - 9 } & SNR (dB) & SNR & MSE & Time & SNR (dB) & SNR & MSE & Time \\
\hline Minimaxi-hard & 60.8527 & 1147.3861 & $4.65 \mathrm{E}-05$ & 0.1252 & 64.1432 & 1744.2431 & $4.29 \mathrm{E}-05$ & 0.5066 \\
Minimaxi-Soft & 60.8527 & 1147.3861 & $4.65 \mathrm{E}-05$ & 0.1252 & 64.1432 & 1744.2431 & $4.29 \mathrm{E}-05$ & 0.5066 \\
SUREShrink-Hard & 60.8527 & 1147.3861 & $4.65 \mathrm{E}-05$ & 0.0944 & 64.1432 & 1492.6542 & $4.29 \mathrm{E}-05$ & 0.1647 \\
SUREShrink-Soft & 60.8527 & 1147.3861 & $4.65 \mathrm{E}-05$ & 0.0944 & 64.1432 & 1492.6542 & $4.29 \mathrm{E}-05$ & 0.1647 \\
ATMDe & $\mathbf{6 7 . 3 3 5 5}$ & $\mathbf{2 4 6 1 . 1 6 6 7}$ & $\mathbf{1 . 8 8 E - 0 5}$ & $\mathbf{0 . 0 8 4 5}$ & $\mathbf{6 5 . 1 0 8 4}$ & $\mathbf{1 9 7 0 . 6 9 5 2}$ & $\mathbf{3 . 5 3 E - 0 5}$ & $\mathbf{0 . 1 0 2 7}$ \\
\hline
\end{tabular}

We conclude that, for ATMDe with other standard wavelet in [28], see in Table 4, the ATMDe is the highest. The indoor signals of it are higher than other standard wavelet with SNR 114.50\%, MSE 147.34\%, and the processing time $11.72 \%$. Similarly, the outdoor signals of it are higher than with SNR $32.03 \%$, MSE $21.53 \%$, and the processing time $60.37 \%$. Thus, the new proposed is suitable in noisy speech and noisy environment.

Table 5. Example 3: Signal to Noise Ratio (SNR): Comparison of ATMDe with other standard wavelet in Noisy environment with my recorded.

\begin{tabular}{l|cccc|cccc}
\hline \multirow{2}{*}{ Signal } & \multicolumn{4}{|c|}{ Indoor } & \multicolumn{3}{c}{ Outdoor } \\
\cline { 2 - 10 } & SNR (dB) & SNR & MSE & Time & SNR (dB) & SNR & MSE & Time \\
\hline Minimaxi-hard & 69.0661 & 2840.6667 & $4.82 \mathrm{E}-05$ & 0.4088 & 62.9282 & 1826.8410 & $2.26 \mathrm{E}-04$ & 0.4078 \\
\hline Minimaxi-Soft & 69.1961 & 2885.1000 & $4.63 \mathrm{E}-05$ & 0.3778 & 62.9282 & 1826.8410 & $2.26 \mathrm{E}-04$ & 0.4078 \\
\hline SUREShrink-Hard & 69.0661 & 2840.6667 & $4.82 \mathrm{E}-05$ & 0.4626 & 62.9282 & 1826.8410 & $2.26 \mathrm{E}-04$ & 0.4854 \\
\hline SUREShrink-Soft & 69.0661 & 2840.6667 & $4.82 \mathrm{E}-05$ & 0.4626 & 62.9282 & 1826.8410 & $2.26 \mathrm{E}-04$ & 0.4854 \\
\hline ATMDe & $\mathbf{6 9 . 5 6 5 5}$ & $\mathbf{3 0 0 8 . 7 0 0 0}$ & $\mathbf{4 . 3 1 E - 0 5}$ & $\mathbf{0 . 3 3 9 4}$ & $\mathbf{6 5 . 0 6 8 8}$ & $\mathbf{2 3 5 0 . 9 0 7 1}$ & $\mathbf{1 . 4 3 E - 0 4}$ & $\mathbf{0 . 3 4 7 0}$ \\
\hline
\end{tabular}

The results in the ATMDe with other standard wavelet in noisy environment are summarized in the example 3 in Table 5. It is highest of indoor signal with SNR 5.58\%, MSE $11.83 \%$, and the processing time $36.30 \%$. Likewise, the outdoor is more than $28.68 \%$, MSE $58.04 \%$, and the processing time $39.88 \%$

Moreover, Table 6 and Table 7 show the results of the effectiveness of the proposed method. Two set of additive noise experiments were added to the sentences on [27] for Table 6 and Table 7 is used data in section 2.2 at SNR levels of $-10,-5,0,+5$, and $+10 \mathrm{~dB}$ in type noisy environment indoor and outdoor. SNR (dB), SNR, and MSE results for experiments. They compared noisy speech of SNR input and SNR output for different denoising methods. In the first is shown in Table 6 and example 4 and in the second is shown in Table 7 and example 5. The overall result indicated the SNR (dB), SNR, and MSE of the SNR output (ATMDe algorithm) are given the best results of SNR input for all SNR levels.

Table 6. Example 4: Signal to Noise Ratio (SNR): Comparison input SNR versus output SNR at SNR levels of $-10,-5,0,+5$, and +10 .

\begin{tabular}{|c|c|c|c|c|c|c|c|}
\hline & \multirow{2}{*}{\multicolumn{2}{|c|}{ Noisy speech }} & \multicolumn{5}{|c|}{ Add white Gaussian noise with SNR level } \\
\hline & & & -10 & -5 & 0 & 5 & 10 \\
\hline \multirow{6}{*}{ Indoor } & \multirow{3}{*}{ SNR input } & SNR (dB) & 29.2752 & 34.0374 & 40.7413 & 46.4345 & 52.0855 \\
\hline & & SNR & 29.3452 & 50.6381 & 110.1301 & 214.1192 & 410.1180 \\
\hline & & MSE & 0.0328 & 0.0104 & 0.0033 & 0.0010 & 0.0003 \\
\hline & \multirow{3}{*}{ SNR output } & SNR (dB) & 31.3724 & 37.2333 & 43.5070 & 48.9527 & 53.7771 \\
\hline & & SNR & 37.2323 & 73.2316 & 151.2141 & 286.5677 & 500.4168 \\
\hline & & MSE & 0.0191 & 0.0061 & 0.0019 & 0.0006 & 0.0002 \\
\hline \multirow{6}{*}{ Outdoor } & \multirow{3}{*}{ SNR input } & SNR $(\mathrm{dB})$ & 30.9983 & 37.1992 & 44.5451 & 50.6803 & 56.1953 \\
\hline & & SNR & 36.3315 & 74.7263 & 173.6417 & 354.5826 & 672.6639 \\
\hline & & MSE & 0.0222 & 0.0070 & 0.0022 & 0.0007 & 0.0002 \\
\hline & \multirow{3}{*}{ SNR output } & SNR (dB) & 34.0955 & 40.4895 & 47.4398 & 53.0152 & 57.7869 \\
\hline & & SNR & 51.9930 & 109.2614 & 242.5972 & 465.1556 & 813.0302 \\
\hline & & MSE & 0.0130 & 0.0041 & 0.0013 & 0.0004 & 0.0002 \\
\hline
\end{tabular}


In Table 6, the results show of indoor and outdoor that SNR output more high SNR dB, SNR, and MSE than SNR input. The results shown in Table 6 are given the spectrogram of noisy speech and denoised speech. Figures 7(a), 7(c), 8(a), and 8(c) represented noisy speech adding white Gaussian noise at SNR $-5 \mathrm{~dB}$ and 5 dB, and Figs. 7(b), 7(d), 8(b), and 8(d) represented denoised speech of them. The results show that the effects of the noise can be seen in the high spectrogram. The denoising reduced the energy noise.

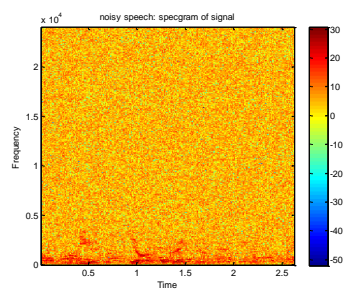

(a)

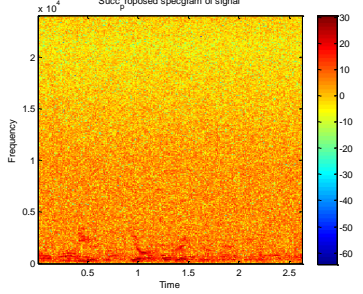

(b)

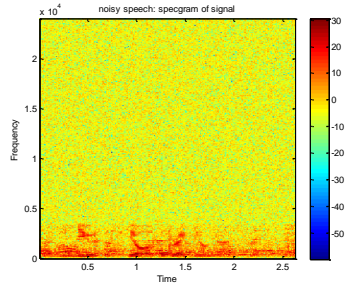

(c)

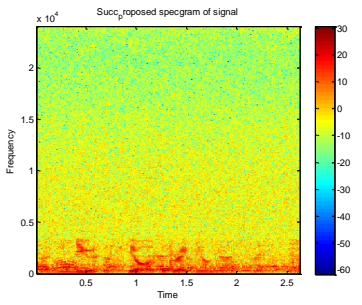

(d)

Fig. 7. Comparison of the results at $\mathrm{SNR}+/-5 \mathrm{~dB}$ on noisy speech of indoor: (a) Original signal adding white Gaussian noise at SNR -5 dB, (b) denoised speech at SNR -5 dB, (c) Noisy speech adding white Gaussian noise at SNR $5 \mathrm{~dB}$, and (d) denoised speech at SNR $5 \mathrm{~dB}$.

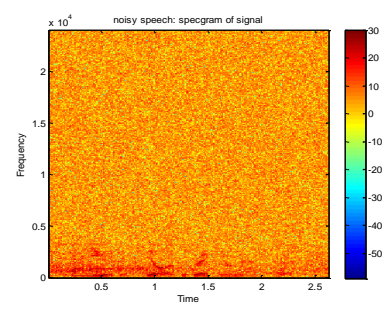

(a)

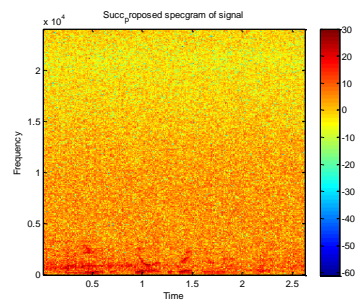

(b)

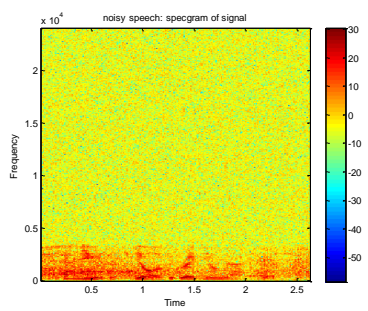

(c)

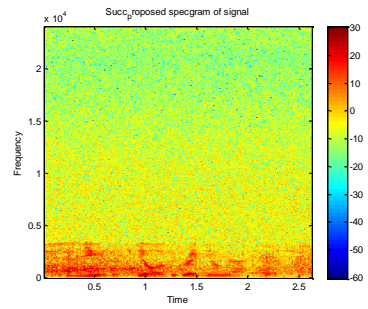

(d)

Fig. 8. Comparison of the results at $\mathrm{SNR}+/-5 \mathrm{~dB}$ on noisy speech of outdoor: (a) Original signal adding white Gaussian noise at SNR $-5 \mathrm{~dB}$, (b) denoised speech at SNR -5 dB, (c) Noisy speech adding white Gaussian noise at SNR $5 \mathrm{~dB}$, and (d) denoised speech at SNR $5 \mathrm{~dB}$.

Table 7. Example 5: Signal to Noise Ratio (SNR): Comparison of SNR of noisy speech input versus SNR of ATMDe with my recorded.

\begin{tabular}{|c|c|c|c|c|c|c|c|}
\hline \multirow{2}{*}{\multicolumn{3}{|c|}{ Noisy speech }} & \multicolumn{5}{|c|}{ Add white Gaussian noise with SNR level } \\
\hline & & & -10 & -5 & 0 & 5 & 10 \\
\hline \multirow{6}{*}{ Indoor } & \multirow{3}{*}{ SNR input } & SNR (dB) & 37.5956 & 45.4148 & 53.0654 & 59.4037 & 65.1374 \\
\hline & & SNR & 75.9235 & 186.5317 & 450.2372 & 934.0089 & 1807.6000 \\
\hline & & MSE & 0.0104 & 0.0033 & 0.0010 & 0.0003 & 0.0001 \\
\hline & \multirow{3}{*}{ SNR output } & SNR $(\mathrm{dB})$ & 41.5457 & 49.1688 & 56.1484 & 61.6463 & 65.7831 \\
\hline & & SNR & 119.6174 & 287.4399 & 642.4813 & 1209.9000 & 1949.6667 \\
\hline & & MSE & 0.0061 & 0.0019 & 0.0006 & 0.0002 & 0.0001 \\
\hline \multirow{6}{*}{ Outdoor } & \multirow{3}{*}{ SNR input } & SNR $(\mathrm{dB})$ & 32.5225 & 40.0356 & 47.3694 & 53.7444 & 59.4224 \\
\hline & & SNR & 48.9487 & 121.2002 & 288.8133 & 610.8006 & 1191.6037 \\
\hline & & MSE & 0.0429 & 0.0136 & 0.0043 & 0.0014 & 0.0004 \\
\hline & \multirow{3}{*}{ SNR output } & SNR (dB) & 36.0688 & 43.6746 & 50.3509 & 55.9274 & 60.3086 \\
\hline & & SNR & 74.3062 & 185.0065 & 413.3454 & 802.9926 & 1331.2330 \\
\hline & & MSE & 0.0256 & 0.0080 & 0.0026 & 0.0009 & 0.0004 \\
\hline
\end{tabular}

In Table 7, the results show of indoor and outdoor that SNR output more high SNR dB, SNR, and MSE than SNR input. The results shown in Table 7 are given the spectrogram of noisy speech and denoised speech. Figures 9(a), 9(c), 10(a), and 10(c) represented noisy speech adding white Gaussian noise at SNR -5 dB and $5 \mathrm{~dB}$, and Figs. 9(b), 9(d), 10(b), and 10(d) represented denoised speech of them. The result of denoising results in the time-domain show that the effects of the noise can be seen in the high spectrogram. The denoising reduced the energy noise. 


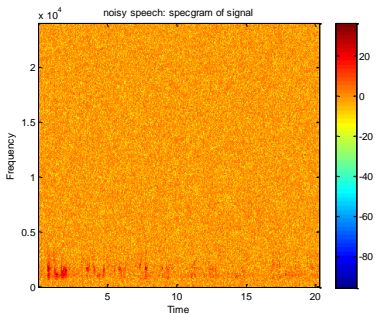

(a)

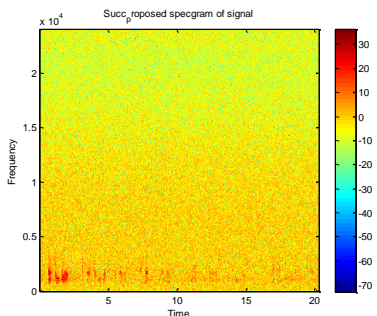

(b)

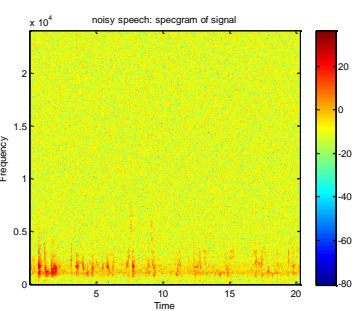

(c)

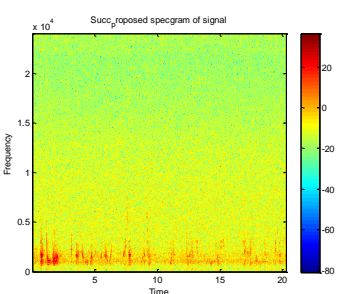

(d)

Fig. 9. Comparison of the results at $\mathrm{SNR}+/-5 \mathrm{~dB}$ on noisy speech of indoor with my recorded: (a) Noisy speech adding white Gaussian noise at SNR $-5 \mathrm{~dB}$, (b) denoised speech at SNR $-5 \mathrm{~dB}$, (c) Noisy speech adding white Gaussian noise at SNR $5 \mathrm{~dB}$, and (d) denoised speech at SNR $5 \mathrm{~dB}$.

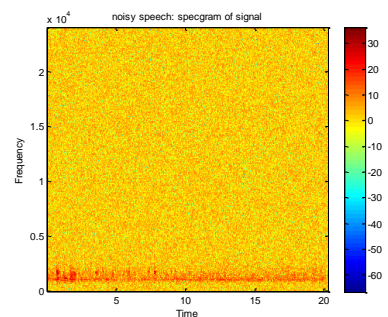

(a)

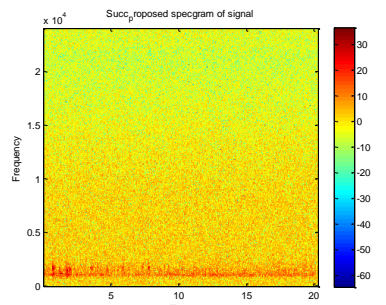

(b)

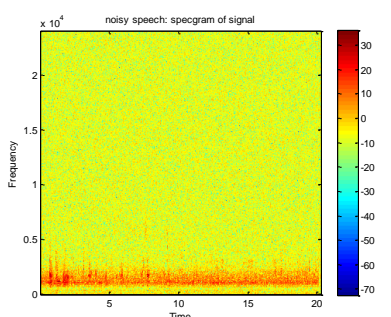

(c)

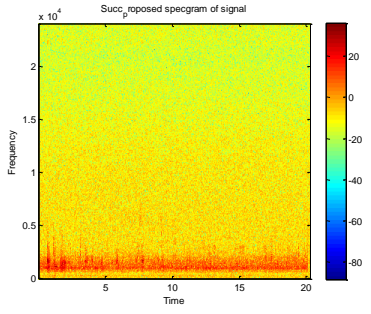

(d)

Fig. 10. Comparison of the results at $\mathrm{SNR}+/-5 \mathrm{~dB}$ on noisy speech of outdoor with my recorded: (a) Noisy speech adding white Gaussian noise at SNR $-5 \mathrm{~dB}$, (b) denoised speech at SNR $-5 \mathrm{~dB}$, (c) Noisy speech adding white Gaussian noise at SNR $5 \mathrm{~dB}$, and (d) denoised speech at SNR $5 \mathrm{~dB}$.

Table 8. Example 6: Signal to Noise Ratio (SNR): Comparison of New Method with other standard denoising method at $2 \mathrm{~dB}$.

\begin{tabular}{lllll}
\hline Method & Blocks & Bumps & Heavy sine & Doppler \\
\hline Minimaxi-hard & 9.1596 & 26.9421 & $\mathbf{5 4 . 1 7 1 6}$ & $\mathbf{1 4 . 0 8 2 8}$ \\
Minimaxi-Soft & 10.0220 & 26.9421 & $\mathbf{5 4 . 1 7 1 6}$ & $\mathbf{1 4 . 0 8 2 8}$ \\
SUREShrink-Hard & 7.9252 & 26.9421 & $\mathbf{5 4 . 1 7 1 6}$ & $\mathbf{1 4 . 0 8 2 8}$ \\
SUREShrink-Soft & 8.3402 & 26.9421 & $\mathbf{5 4 . 1 7 1 6}$ & $\mathbf{1 4 . 0 8 2 8}$ \\
X. Zhang, et al. [26] & $\mathbf{1 3 . 5 8 8 1}$ & 14.2969 & 14.4874 & 13.6232 \\
T.-H. Yi, et al. [29] & 13.0926 & 13.6467 & 18.9648 & 13.4134 \\
ATMDe & 9.9586 & $\mathbf{2 6 . 9 5 3 6}$ & 54.1543 & 14.0804 \\
\hline
\end{tabular}

The example 6 in Table 8 is used four well-known benchmark signals, including Block, Bumps, Heavy sine and Doppler of signal synthesis with the noisy signals on $2 \mathrm{~dB}$ with sample points of signal are 2048 [26]. These results shown in above are compared using SNR. The best results are indicated by bold-face. It can be seen that the new method is high SNR than other denoising method in bumps.

The results of ATMDe show that it is highest SNR in various types of environmental noise and speech without noise, moreover, it is highest SNR in Bumps that is signal synthesis with Bumps but the ATMDe is the second highest SNR in another signal synthesis with Heavy sine, Doppler. 


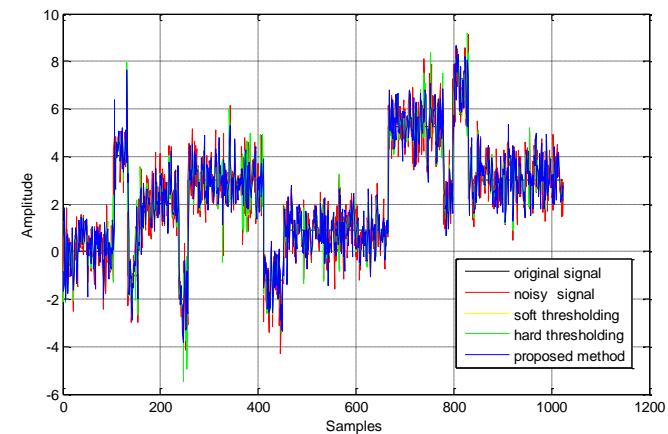

(a)

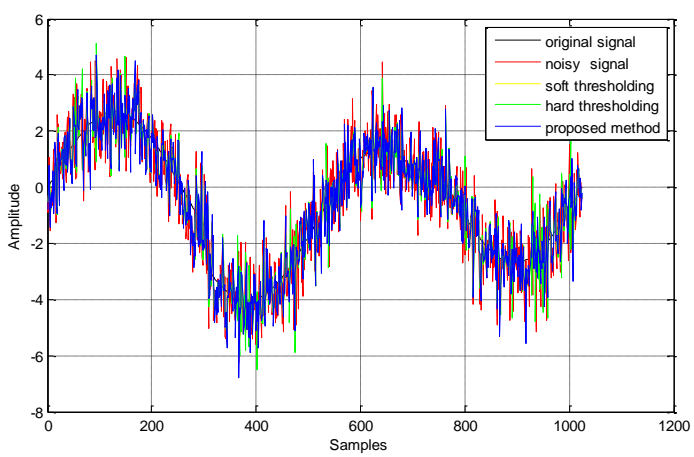

(c)

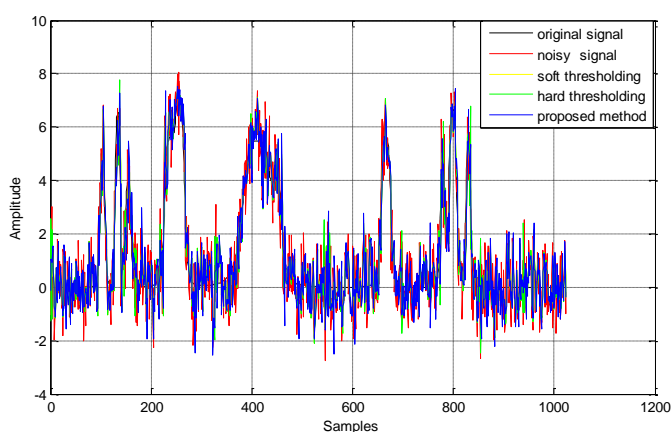

(b)

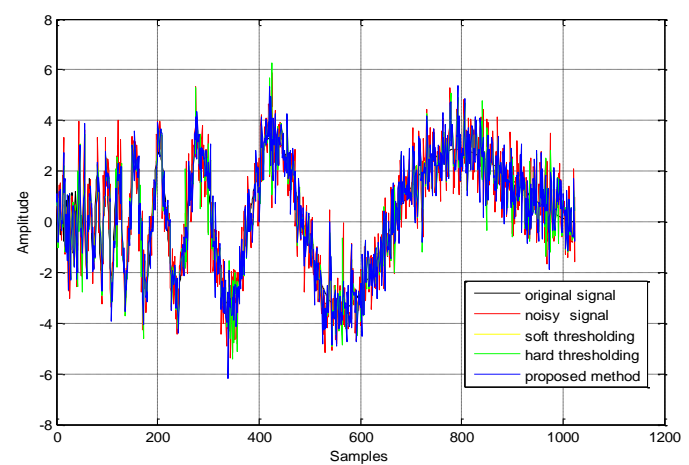

(d)

Fig. 11. Comparison of different thresholding schemes with Benchmark signals denosing at $2 \mathrm{~dB}$ : (a) Blocks signal; (b) Bumps signal; (c) Heavy sine signal; (d) Doppler signal.

The results of the four benchmark signals at $2 \mathrm{~dB}$ are showed in Fig. 11 for compare the results form denoising method in time domain as example 6 in Table 8. The threshold values of soft and hard thresholding used Minimaxi method because the SNR of it is higher than SUREShrink method but the threshold values of proposed method is used adaptive threshold of ATMDe.

\section{Conclusion}

A new method for noisy speech using 1-D wavalet has been presented called Adaptive Thresholding with Mean for hybrid Denoising method of hard and soft function (ATMDe). This method is based on wavelet denoising methods with Discrete wavelet transform with families Coiflet1 because of the results derived from experimental, decomposition with level 1 because low complexity, and it represented adaptive thresholding of subband called ISSUE 1 and new denoised wavelet coefficients called ISSUE 2 that the results show that new proposed is able to effectively denoise. There are two advantages of the proposed method: First, it provides each sub-band as an independent thresholding for denoising. Second, the new method can be used to minimize time of computation that low complexity.

The performance of the new method using speech corpus, real signals, four well-known benchmark signals, and signals adding white Gaussian noise at different SNR levels $-10,-5,0,+5$, and $+10 \mathrm{~dB}$. The result found that the denoising reduced the energy noise. The result of new method with SNR and MSE is better than standard denoising methods.

Future research will be improve the performance and apply this technique in the gain of in hearing loss and hearing aids 


\section{References}

[1] S. V. Vaseghi, Advanced Digital Signal Processing and Noise Reduction, 4th ed. John Wiley \& Sons, 2008.

[2] M. T. Johnson, X. Yuan, and Y. Ren, "Speech signal enhancement through adaptive wavelet thresholding," Speech Communication, vol. 49, no. 2, pp. 123-133, 2007.

[3] S. Mallat, $A$ Wavelet Tour of Signal Processing The Sparse Way, 3rd ed. Elsevier, 2009.

[4] V. Hamacher, J. Chalupper, J. Eggers, E. Fischer, U. Kornagel, H. Puder, and U. Rass, "Signal processing in high-end hearing aids: State of the art, challenges, and future trends," EURASIP Journal on Applied Sig nal Processing, vol. 2005, no. 18, pp. 2915-2929, 2005.

[5] S. Jangjit and M. Ketcham, "A survey of hearing loss and hearing aids," in Int'l Conference on Advanced Computational Technologies \& Creative Media (ICACTCM'2014), Pattaya (Thailand), 2014.

[6] C. Dimoulas, G. Kalliris, G. Papanikolaou, and A. Kalampakas, "Novel wavelet domain Wiener filtering de-noising techniques: Application to bowel sounds captured by means of abdominal surface vibrations," Biomedical Signal Processing and Control, vol. 1, no. 3, pp. 177-218, 2006.

[7] L. Zhang and X.-H. Yang, "The application of an improved wavelet threshold denoising method in heart sound signal," in Cross Strait Quad-Regional Radio Science and Wireless Technology Conference (CSQRWC), 2011, 2011, pp. 1115-1117.

[8] Y. Ghanbari and M. R. Karami-Mollaei, "A new approach for speech enhancement based on the adaptive thresholding of the wavelet packets," Speech Communication, vol. 48, pp. 927-940, 2006.

[9] M. Srivastava, C. L. Anderson, and J. H. Freed, "A new wavelet denoising method for selecting decomposition levels and noise thresholds," IEEE Access, vol. 4, pp. 3862-3877, 2016.

[10] Y. Lin and J. Cai, "A new threshold function for signal denoising based on wavelet transform," in 2010 International Conference on Measuring Technology and Mechatronics Automation, 2010, pp. 200-203.

[11] C.-T. Lu and H.-C. Wang, "Enhancement of single channel speech based on masking property and wavelet transform," Speech Communication, vol. 41, pp. 409-427, 2003.

[12] C. E. Martínez, J. Goddard, L. E. Di Persia, D. H. Milone, and H. L. Rufiner, "Denoising sound signals in a bioinspired non-negative spectro-temporal domain," Digital Signal Processing, vol. 38, pp. 22-31, 2015.

[13] H. Krim, D. Tucker, S. Mallat, and D. Donoho, "On denoising and best signal representation," IEEE Transactions on Information Theory, vol. 45, pp. 2225-2238, 1999.

[14] D. L. Donoho, "De-noising by soft-thresholding," IEEE Transactions on Information Theory, vol. 41, pp. 613-627, 1995.

[15] A. Sangwan, M. C. Chiranth, H. S. Jamadagni, R. Sah, R.V. Prasad, and V. Gaurav, "VAD techniques for real-time speech transmission on the Internet," in High Speed Networks and Multimedia Communications 5th IEEE International Conference on, 2002, pp. 46-50.

[16] M. Marzinzik and B. Kollmeier, "Speech pause detection for noise spectrum estimation by tracking power envelope dynamics," IEEE Transactions on Speech and Audio Processing, vol. 10, pp. 109-118, 2002.

[17] R. Narayanam, "Efficient de-noising performance of a combined algorithm of translation invariant (TI) wavelets and independent component analysis over TI wavelets for speech-auditory brainstem responses," Procedia Computer Science, vol. 54, pp. 829-837, 2015.

[18] M. A. Kabir and C. Shahnaz, "Denoising of ECG signals based on noise reduction algorithms in EMD and wavelet domains," Biomedical Signal Processing and Control, vol. 7, pp. 481-489, 2012.

[19] K. Likitsupin, P. Punyabukkana, C. Wutiwiwatchai, and A. Suchato, "Acoustic-phonetic approaches for improving segment-based speech recognition for large vocabulary continuous speech," Engineering Journal, vol. 20, no. 2, pp. 179-197, 2016.

[20] K. Yang, Z. He, W. Yang, Q. Tang, D. Li, Z. Wang, Q. Lin, and W. Jia, "Heart sound denoising using computational auditory scene analysis for a wearable stethoscope," in 2013 IEEE 56th International Midwest Symposium on Circuits and Systems (MWSCAS), 2013, pp. 1220-1223.

[21] D. Emmanouilidou, E. D. McCollum, D. E. Park, and M. Elhilali, "Adaptive noise suppression of pediatric lung auscultations with real applications to noisy clinical settings in developing countries," IEEE Transactions on Biomedical Engineering, vol. 62, pp. 2279-2288, 2015.

[22] J. Rafiee, M. A. Rafiee, N. Prause, and M. P. Schoen, "Wavelet basis functions in biomedical signal processing," Expert Systems with Applications, vol. 38, pp. 6190-6201, 2011.

[23] A. Assi, Engineering Education and Research Using MATLAB. InTech, 2011, pp. 26-53.

[24] D. L. Donoho and J. M. Johnstone, "Ideal spatial adaptation by wavelet shrinkage," Biometrika, vol. 81, pp. 425-455, 1994. 
[25] M. Bahoura and J. Rouat, "Wavelet speech enhancement based on the Teager energy operator," IEEE Signal Processing Letters, vol. 8, pp. 10-12, 2001.

[26] X. Zhang, J. Li, J. Xing, P. Wang, and D. Fu, "A kent chaos artificial bee colony algorithm based wavelet thresholding method for signal denoising," in 2016 12th World Congress on Intelligent Control and Automation (WCICA), 2016, pp. 3129-3134.

[27] P. Loizou. (2016, June 2). NOIZEUS: A Noisy Speech Corpus for Evaluation of Speech Enhancement Algorithms. [Online]. Available: http://ecs.utdallas.edu/loizou/speech/noizeus/

[28] M. A. Oktar, M. Nibouche, and Y. Baltaci, "Denoising speech by notch filter and wavelet thresholding in real time," in 2016 24th Signal Processing and Communication Application Conference (SIU), 2016, pp. 813816.

[29] T. H. Yi, H. N. Li, and X. Y. Zhao, "Noise smoothing for structural vibration test signals using an improved wavelet thresholding technique," Sensors (Basel, Switzerland), vol. 12, no. 8, pp. 11205-11220, 2012. 